\begin{tabular}{|c|l|}
\hline Title & Critical cluster size of InA s quantum dots formed by Stranski-Krastanow mode \\
\hline Author(s) & Shiramine, Ken-ichi; Itoh, Tomohiko; Muto, Shunichi \\
\hline Citation & $\begin{array}{l}\text { Journal of Vacuum Science \& Technology B: Microelectronics and Nanometer Structures, 22(2), 642-646 } \\
\text { https:/doi.org/L0.1116/1.1651113 }\end{array}$ \\
\hline Issue Date & 200403 \\
\hline Doc URL & http://hdl.handle.net/2115/5857 \\
\hline Type & article \\
\hline File Information & JVS\&TB.pdf \\
\hline
\end{tabular}

Instructions for use 


\title{
Critical cluster size of InAs quantum dots formed by Stranski-Krastanow mode
}

\author{
Ken-ichi Shiramine, ${ }^{\text {a) }}$ Tomohiko Itoh, and Shunichi Muto \\ Department of Applied Physics, Hokkaido University, Sapporo 060-8628, Japan
}

(Received 8 July 2003; accepted 5 January 2004; published 10 March 2004)

\begin{abstract}
The number of In atoms in a critical cluster, $i^{*}$, in Stranski-Krastanow (S-K) mode of InAs islands was determined to be $1-10$. The $i^{*}$ was determined using an activation energy $E_{A}$ of $2.0 \mathrm{eV}$ determined from an Arrhenius plot of the saturated density of InAs islands formed on a GaAs (001) surface by $\mathrm{S}-\mathrm{K}$ mode of molecular beam epitaxy [K. Shiramine et al., J. Cryst. Growth 242, 332 (2002)], and an activation energy of $1.6 \mathrm{eV}$ for migration (surface diffusion) of In adatoms, inferred from other references. The common value $\sim 2.0 \mathrm{eV}$ of $E_{A}$ in $\mathrm{S}-\mathrm{K}$ mode was ascribed to the small $i^{*}$. (ㄷ) 2004 American Vacuum Society. [DOI: 10.1116/1.1651113]
\end{abstract}

\section{INTRODUCTION}

In Stranski-Krastanow ( $\mathrm{S}-\mathrm{K})$ mode in molecular beam epitaxy (MBE), the initial two-dimensional (2D) growth (layer growth) and subsequent 3D growth (island growth) produce a wetting layer and islands, respectively. ${ }^{1}$ The islands are coherent and defect free. They are regarded as selforganized quantum dots because they show quantum-size effects. Therefore, they are expected to yield novel optical and electronic devices. The growth of 2 monolayers (MLs) of InAs on GaAs, discussed in the present article, is a typical $\mathrm{S}-\mathrm{K}$ mode.

In the present study, we will determine the critical cluster size in $\mathrm{S}-\mathrm{K}$ mode. The critical cluster is important in the nucleation of crystal growth; the formation of a critical cluster is assumed to be the nucleation of an island ("cluster" is used as a synonym of "island"). The concept of critical clusters in the capillarity model, ${ }^{2}$ in which surface energy is considered, can be explained as shown in Fig. 1, where the total free energy $G$ of a 3D island is given as a function of its radius $r$. $G$ increases with increasing $r$ for $r<r^{*}$ and decreases for $r>r^{*}$. A critical cluster is an island for which $r$ is $r^{*}$, while metastable and stable clusters are islands for which $r<r^{*}$ and $r>r^{*}$, respectively. Metastable clusters tend to disappear because becoming smaller is energetically favorable. On the other hand, stable clusters tend to grow larger because growing larger is energetically favorable. Almost all the islands that grow beyond a critical cluster grow larger. The critical cluster size $i^{*}$, i.e., the number of atoms which compose a critical cluster, dominates nucleation. In spite of this, only a few reports in which $i^{*}$ for $\mathrm{S}-\mathrm{K}$ mode is discussed quantitatively have been published. ${ }^{3,4}$ Here we will determine $i^{*}$ in $\mathrm{S}-\mathrm{K}$ mode from the activation energy, reported already, determined from an Arrhenius plot of the saturated density of islands.

The authors have already reported the saturated density of InAs $\mathrm{S}-\mathrm{K}$ islands as a function of growth temperature $T_{S} .5$ The activation energy $E_{A}$ determined from the Arrhenius plot of the density was $2.0 \mathrm{eV}$, where the saturated density $N_{s}$ is expressed as $N_{s} \propto \exp \left[E_{A} /\left(k_{B} T_{s}\right)\right]$ and $k_{B}$ is the Boltzmann

${ }^{a)}$ Electronic mail: shira@eng.hokudai.ac.jp constant. Values of $E_{A}$ for $\mathrm{S}-\mathrm{K}$ mode reported in other article $^{3,6-12}$ are also $\sim 2.0 \mathrm{eV}$, as pointed out in Ref. 5, although the origin of this common $E_{A}$ has yet to be explained. Here we will analyze the same experimental results using a more precise theory to elucidate the origin of the common $E_{A}$.

In the present article, $i^{*}$ and the binding energy of a critical cluster, $E_{i *}$, is discussed based on $E_{A}$ of $2.0 \mathrm{eV}$ and the activation energy for adatom migration (surface diffusion), $E_{\text {diff }}$, of $1.6 \mathrm{eV}$. $E_{\text {diff }}$ of $1.6 \mathrm{eV}$ for In adatoms is inferred from experimental data for $\mathrm{Ga}$ adatoms. The relationship between $i^{*}$ and $E_{i *}$ is obtained from $E_{A}$ and $E_{\text {diff }}$ using Frankl and Venables' theory. ${ }^{13}$ We conclude that $i^{*}$ is $1-10$ (In atoms only). The common value $\sim 2.0 \mathrm{eV}$ of $E_{A}$ for $\mathrm{S}-\mathrm{K}$ islands is ascribed to the small $i^{*}$.

\section{EXPERIMENTAL AND RESULTS}

Crystal growth was carried out by MBE. 2 monolayers of InAs were grown on a GaAs (001) substrate at growth temperatures $T_{s}$ of $450-530^{\circ} \mathrm{C}$. The surface was observed by atomic force microscopy (AFM) in air. The area density of the InAs $\mathrm{S}-\mathrm{K}$ islands was determined from AFM images as a function of $T_{s}$. Details of the experiments have been published elsewhere. ${ }^{5}$

Figure 2 shows an Arrhenius plot of the island density obtained. ${ }^{5}$ The density decreases with an increase in $T_{s}$. The decrease is produced by an increase in the diffusion length of adatoms with $T_{S} \cdot{ }^{5-12}$ The activation energy $E_{A}$ determined from the Arrhenius plot was $2.0 \mathrm{eV}$ from the slope of the fitted line (Fig. 2). The results agree with those of other studies on $\mathrm{S}-\mathrm{K}$ islands: ${ }^{3,6-11}$ the density decreases with an increase in $T_{s}$, and $E_{A}$ is $\sim 2.0 \mathrm{eV} .^{5,14}$

\section{DISCUSSION}

\section{A. Expression for saturated density of islands}

We discuss the expression for the saturated density of islands. Islands of metals formed by vacuum evaporation were the focus of study in the $1960 \mathrm{~s}$ and $1970 \mathrm{~s} .{ }^{15}$ Theories on the nucleation of metal islands published in those days 


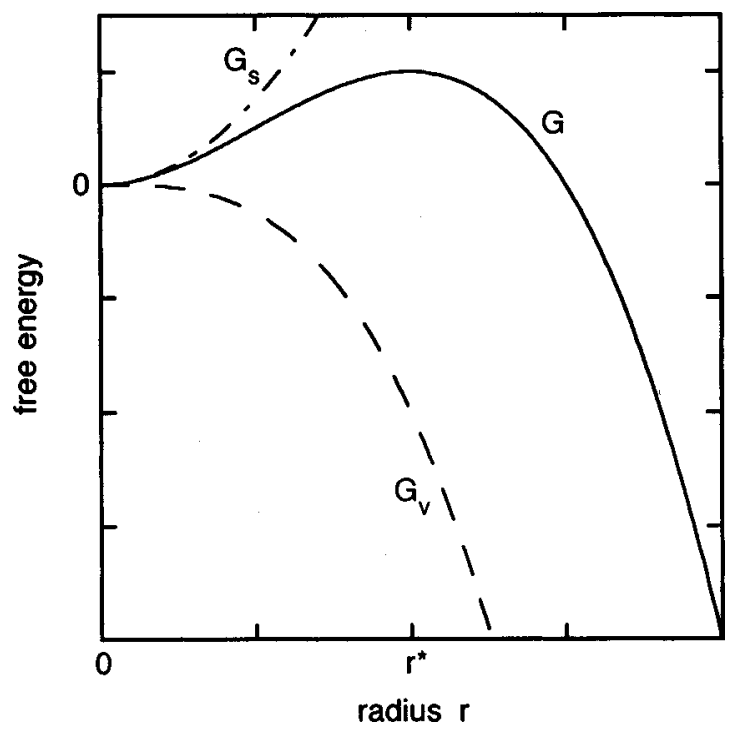

FIG. 1. Schematic graph of the total free energy $G$, the volume free energy $G_{v}$, and the surface free energy $G_{s}$ of a 3D island as a function of its radius $r$, where $G=G_{v}+G_{s}, G_{v} \propto-r^{3}$, and $G_{s} \propto r^{2}$.

can be applied to $\mathrm{S}-\mathrm{K}$ islands. Frankl and Venables have considered the saturation of island density caused by the depletion of adatoms $;^{13}$ we conclude that their theory is appropriate for use in the analysis of the nucleation of $\mathrm{S}-\mathrm{K}$ islands.

The nucleation theory gives us an expression for the saturated density of islands; we should consider the origin of the saturation of island density to determine whether the theory is applicable to $\mathrm{S}-\mathrm{K}$ mode or not. The saturation in the theories is caused by: (i) the coalescence of islands and (ii) the depletion of adatoms. A density saturation of $\mathrm{S}-\mathrm{K}$ islands is believed to be caused by an adatom depletion due to the capture of adatoms by islands. Here we have not considered the possibility of saturation by coalescence. Indeed, few large incoherent islands are observed in 2 ML growth of InAs on GaAs although the density is saturated.

Many research groups have suggested expressions for the saturated island density. Here we consider only complete condensation, in which all incident atoms contribute to growth and no atoms desorb from the surface, because growth conditions of MBE are usually set so that complete condensation occurs. Stowell and Hutchinson have shown the expression $N_{s} \propto \exp \left\{\left(E_{i^{*}}+i^{*} E_{\mathrm{diff}}\right) /\left[\left(i^{*}+\frac{5}{2}\right) k_{B} T_{s}\right]\right\}$ for the saturation caused by the coalescence of $3 \mathrm{D}$ islands and the expression $N_{s} \propto \exp \left\{\left(E_{i^{*}}+i^{*} E_{\mathrm{diff}}\right) /\left[\left(i^{*}+2\right) k_{B} T_{s}\right]\right\}$ for the saturation caused by the coalescence of $2 \mathrm{D}$ islands. ${ }^{16,17}$ These expressions cannot be applied to $\mathrm{S}-\mathrm{K}$ islands because they are for the saturation due to coalescence.

Frankl and Venables have suggested an expression using island density for the saturation caused by the depletion of adatoms; ${ }^{13}$ in the saturation caused by depletion, incident atoms are caught by existing islands and do not nucleate. They deduced the expression by solving rate equations for an island density under the following assumptions: (i) an incident atom must condense, and it is never desorbed from the

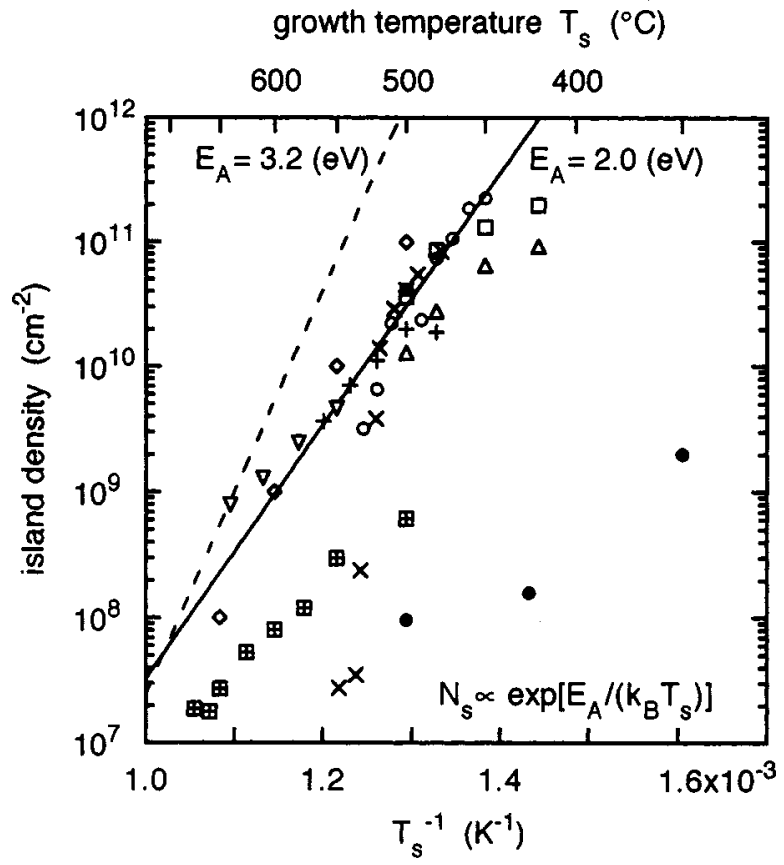

FIG. 2. Arrhenius plot of S-K island density: (O) 2 ML InAs/GaAs (Ref. 5);

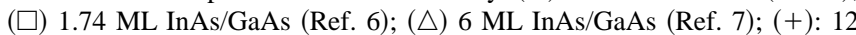
ML $\mathrm{In}_{0.6} \mathrm{Ga}_{0.4} \mathrm{As} / \mathrm{GaAs}$ (Ref. 8); $(\times) \mathrm{GaSb} / \mathrm{GaAs}$ (Ref. 9); ( $\left.\diamond\right) 2.5 \mathrm{ML}$ $\mathrm{In}_{0.5} \mathrm{Ga}_{0.5} \mathrm{As} / \mathrm{GaAs}$ metalorganic chemical vapor deposition (MOCVD) (Ref. $10) ;(\nabla) 3.5 \mathrm{ML} \mathrm{InP} / \mathrm{GaAs} \mathrm{MOCVD}$ (Ref. 11); and $(\boxplus) 10 \mathrm{~nm} \mathrm{InAs} / \mathrm{GaAs}$ MOCVD (Ref. 3). Data for Volmer-Weber islands: (@) $1 \mathrm{ML}$ InAs/GaP (Ref. 12) are also shown for comparison. All data are for MBE except data $(\diamond, \nabla$, and $\boxplus)$ for MOCVD. The solid line is a fitted line for data of $2 \mathrm{ML}$ InAs/GaAs (O). From the slope of the line, activation energy $E_{A}$ was determined to be $2.0 \mathrm{eV}$. Two data points for higher temperature were omitted in the fitting because In desorption was believed to have occured. The broken line shows a slope corresponding to $E_{A}$ of $3.2 \mathrm{eV}$ (see Sec. III D). The figure is a reproduction of Figs. 2 and 3 in Ref. 5. Some data $(X$ and $\boxplus$ ) have been added.

surface; (ii) adatoms are distributed uniformly on the surface; (iii) $i^{*}$ has a constant value; (iv) a stable cluster is absolutely stable and atoms never detach from it; and (v) islands smaller than a critical cluster are in quasi equilibrium, i.e., the size distribution of islands is considered. Frankl and Venables ${ }^{13}$ proposed the expression for saturated density $N_{s}$ :

$$
\begin{aligned}
N_{s}= & {\left[\frac{\sigma_{i *} C_{i *} N_{0}^{2}}{\left(i^{*}+2\right) \sigma_{s}^{i^{*}+2}}\left(\frac{J}{\nu}\right)^{i^{*}+1}\right]^{\left.1 / i^{*}+3\right)} } \\
& \times \exp \left[\frac{E_{i^{*}}+\left(i^{*}+1\right) E_{\mathrm{diff}}}{\left(i^{*}+3\right) k_{B} T_{s}}\right],
\end{aligned}
$$

where $N_{0}$ is the density of adatom sites on the surface, $J$ is a flux of atoms incident on the surface, $\nu$ is the vibration frequency of adatoms, $E_{i *}$ is the binding energy of a critical cluster, i.e., the energy required to decompose a critical cluster into single adatoms ( $E_{i *}$ has a positive value), $C_{i *}$ is a constant given by $C_{i *}=\prod_{j=2}^{i^{*}}\left(\sigma_{j-1} / \sigma_{j}^{\prime}\right)$ (we have $C_{i *}=1$ for $\left.i^{*}=1\right), \sigma_{j}$ is the capture number of a $j$ cluster, $\sigma_{s}$ is the average $\sigma_{j}$ for stable clusters, and $\sigma_{j}^{\prime}$ is the dissociation number of a $j$ cluster. Then, $\sigma_{j}$ is the sum of the probability of jumping into a captured position over all adjacent sites, and $\sigma_{j}^{\prime}$ is the sum of the fraction of possible jump directions 
that carry the atom out of the cluster over the minimally bonded atoms. We can apply Eq. (1) to the Arrhenius plot of the InAs S-K island density (Fig. 2) because the density of islands is saturated at $\sim 2 \mathrm{ML} .{ }^{18}$ Here the number of atoms is defined as the number of In atoms because the motion of group III atoms dominates the growth of III/V compound semiconductors. The assumption of complete condensation is also applied to In atoms only.

Equation (1) was deduced by a more precise theory than that described in the former article: ${ }^{5}$ it was obtained by solving rate equations considering the size distribution of islands [assumption (v)] which was ignored in Ref. 5. In the previous study, the experimental results were analyzed using a model ${ }^{19}$ in which island density is defined by the diffusion length of adatoms. ${ }^{6-12}$ Although the concept of the model is correct for the nucleation of $\mathrm{S}-\mathrm{K}$ islands, at least qualitatively, the conclusions obtained using the model were not satisfactory quantitatively: $E_{\text {diff }}$ obtained was an unexpectedly large value, i.e., $4.0 \mathrm{eV}$. We can expect to obtain a consistent explanation for the same experimental results using Eq. (1).

Stowell deduced the same equation as Eq. (1) except for numerical factors considering depleted regions of adatoms around stable clusters due to capture. ${ }^{20}$ It is noteworthy that Stowell's equation, deduced by assuming depletion, is the same as Eq. (1), deduced by assuming a uniform distribution of adatoms. ${ }^{15}$

\section{B. Applicability of the model to S-K mode}

Here we consider whether we can apply Frankl and Venables' model (Sec. III A) to island nucleation in $\mathrm{S}-\mathrm{K}$ mode of InAs/GaAs growth. We consider: (i) the applicability of the model in which Volmer-Weber mode is assumed to $\mathrm{S}-\mathrm{K}$ mode; (ii) the validity of the assumption that atoms do not detach from stable islands; (iii) the self-size-limiting effect; (iv) the effect of strain; and (v) the effect of interdiffusion between the epilayer and the substrate.

First, the model is applicable to island growth in $\mathrm{S}-\mathrm{K}$ mode. Epitaxial growth by MBE, including island growth in $\mathrm{S}-\mathrm{K}$ mode, can be well described by the picture of adatoms. $^{21}$ In the adatom picture, an incident atom is adsorbed by the surface, migrates on the surface, and is taken into the crystal at a low-energy site, i.e., a step site or a kink site. The behavior of adatoms is well explained in terms of $E_{\text {diff }}$ and $E_{\text {des }}$ in the adatom picture. Frankl and Venables' model fits the adatom picture well. Therefore, the model describes the island growth of $\mathrm{S}-\mathrm{K}$ mode well.

Second, the density obtained under the assumption that atoms do not detach from stable islands is close to the actual value. In actual growth, atoms can detach from stable islands. However, detachment should not markedly affect the density, unless islands disappear. The adatom density may increase because of the detachment of atoms from stable islands, and the change in adatom density may affect the island density. If this is the case, the effect should be small.

Third, the self-size-limiting effect does not affect the density. Although the size of islands is known to be limited automatically in $\mathrm{S}-\mathrm{K}$ mode, the effect occurs only in large islands of which diameter is about $30 \mathrm{~nm}$ or longer. ${ }^{22}$ The effect should not be notable in nucleation because a critical cluster consists only of a few atoms, as discussed later. The adatom density may increase because adatoms cannot attach to large islands due to the self-size-limiting effect; the island density is generally a function of adatom density. However, this does not markedly affect the island density since the self-size-limiting effect occurs after the density is almost saturated because the effect works only on large islands.

Fourth, strain does not affect the density. If nucleation is caused by the assembly of adatoms, as is assumed in the adatom picture, the role of strain is merely to supply lowpotential sites on the tops of islands after nucleation. The tops of islands are relaxed, and the potential energy of an adatom is lower there.

Finally, the effect of interdiffusion between the epilayer and the substrate should be considered. Interdiffusion is known to occur in island growth of $\mathrm{S}-\mathrm{K}$ mode ${ }^{23}$ although the details are unknown. Interdiffusion probably occurs after islands are formed and is not notable in the initial stage of island formation. Hence, the effect of interdiffusion on the density should be small.

\section{Activation energy for migration of adatoms $E_{\text {diff }}$}

We must know $E_{\text {diff }}$ to discuss $i^{*}$ of InAs S-K islands based on Eq. (1). $E_{\text {diff }}$ for an In adatom has not yet been reported. Here, we will infer it from the activation energy $E_{\text {diff }}(\mathrm{Ga})$ for migration of $\mathrm{Ga}$ adatoms on GaAs. We should carefully evaluate reported values of $E_{\text {diff }}(\mathrm{Ga})$ and their determination methods, because various values of $E_{\text {diff }}(\mathrm{Ga})$ have been reported and there is no single widely accepted value. We obtain $E_{\text {diff }}(\mathrm{Ga})$ of $1.8 \mathrm{eV}$ from the following values reported: $\left(E_{\text {des }}(\mathrm{Ga})-E_{\text {diff }}(\mathrm{Ga})\right) / 2$ of $0.34 \mathrm{eV}$ (Ref. $24)$ and $E_{\text {des }}(\mathrm{Ga})$ of $2.5 \mathrm{eV}$ (Ref. 25), where $E_{\text {des }}(\mathrm{Ga})$ is the activation energy for $\mathrm{Ga}$ adatom desorption. As a result, $E_{\text {diff }}$ for In is determined to be $1.6 \mathrm{eV}$.

Shitara and Nishinaga have determined the diffusion length $\lambda_{s}$ of Ga adatoms as a function of $T_{s} \cdot{ }^{24}$ The activation energy of $\lambda_{s}$ was determined to be $0.34 \mathrm{eV}$. The activation energy obtained was $\left(E_{\text {des }}(\mathrm{Ga})-E_{\text {diff }}(\mathrm{Ga})\right) / 2$ because $\lambda_{s}$ is the distance from incidence to desorption of an adatom, i.e., $\quad \lambda_{s}=\sqrt{D_{s} \tau_{s}}=a \exp \left[\left(E_{\mathrm{des}}(\mathrm{Ga})-E_{\mathrm{diff}}(\mathrm{Ga})\right) /\right.$ $\left.\left(2 k_{B} T_{s}\right)\right]$, where $D_{s}$ is the surface diffusion constant $D_{s}$ $=\nu a^{2} \exp \left[-E_{\text {diff }}(\mathrm{Ga}) /\left(k_{B} T_{s}\right)\right], \tau_{s}$ is the resident time of an adatom on the surface $\tau_{s}=\nu^{-1} \exp \left[E_{\mathrm{des}}(\mathrm{Ga}) /\left(k_{B} T_{s}\right)\right]$, and $a$ is the distance between nearest-neighbor sites of adatoms.

Sugiyama et al. determined $E_{\text {des }}(\mathrm{Ga})$ from the growth rate of GaAs as a function of $T_{s} .{ }^{25}$ The activation energy of sublimation of a $\mathrm{Ga}$ atom from GaAs was determined to be $4.9 \mathrm{eV}$, and the activation energy for the desorption of an excess $\mathrm{Ga}$ atom from the GaAs surface was determined to be $2.5 \mathrm{eV}$.

Here we use the activation energy of $2.5 \mathrm{eV}$ reported by Sugiyama et ll. $^{25}$ as $E_{\text {des }}(\mathrm{Ga})$. Values of the activation energy for the sublimation of GaAs, reported to date, ${ }^{26-28}$ fall in the vicinity of one of two values, i.e., $\sim 2.5$ and $\sim 4.9 \mathrm{eV}$. The heat of sublimation of GaAs with the production of $\mathrm{As}_{2}$ 
TABLE I. Heat of reaction obtained from the enthalpy of formation at 298.15 $\mathrm{K}$ and $100 \mathrm{kPa}$ in Refs. 29 and 30. All reactions are endothermic. "(s)" and "(g)" mean solid and gas, respectively, and $\operatorname{In}(\mathrm{g})$ and $\mathrm{As}(\mathrm{g})$ mean monoatomic gas.

\begin{tabular}{ccc}
\hline \hline & \multicolumn{2}{c}{ Heat of reaction } \\
\cline { 2 - 3 } Reaction & $\left(\mathrm{kJ} \mathrm{mol}^{-1}\right)$ & $(\mathrm{eV})$ \\
\hline $\mathrm{Ga}(\mathrm{s}) \rightarrow \mathrm{Ga}(\mathrm{g})$ & 272.0 & 2.819 \\
$\mathrm{GaAs}(\mathrm{s}) \rightarrow \mathrm{Ga}(\mathrm{g})+\frac{1}{2} \mathrm{As}_{2}(\mathrm{~g})$ & 449.0 & 4.654 \\
$\mathrm{GaAs}(\mathrm{s}) \rightarrow \mathrm{Ga}(\mathrm{g})+\frac{1}{4} \mathrm{As}_{4}(\mathrm{~g})$ & 395.1 & 4.095 \\
$\mathrm{GaAs}(\mathrm{s}) \rightarrow \mathrm{Ga}(\mathrm{g})+\mathrm{As}(\mathrm{g})$ & 640.2 & 6.636 \\
$\mathrm{InAs}(\mathrm{s}) \rightarrow \mathrm{In}(\mathrm{g})+\mathrm{As}(\mathrm{g})$ & 585.0 & 6.064 \\
$\mathrm{InAs}(\mathrm{s}) \rightarrow \mathrm{In}(\mathrm{s})+\frac{1}{2} \mathrm{As}_{2}(\mathrm{~g})$ & 153.1 & 1.587 \\
\hline \hline
\end{tabular}

is $4.7 \mathrm{eV}$ and that with the production of $\mathrm{As}_{4}$ is $4.1 \mathrm{eV}$ (Table I). The activation energy of $2.5 \mathrm{eV}$ can be ascribed to the sublimation of a $\mathrm{Ga}$ adatom, and that of $4.9 \mathrm{eV}$ can be ascribed to the sublimation of GaAs with the production of $\mathrm{As}_{2}$ (we can assume the activation energy for sublimation to be the same as the heat of sublimation).

Now we can determine $E_{\text {diff }}$. We obtained $E_{\text {diff }}(\mathrm{Ga})$ of $1.8 \mathrm{eV}$ from the $\left(E_{\text {des }}(\mathrm{Ga})-E_{\text {diff }}(\mathrm{Ga})\right) / 2$ of $0.34 \mathrm{eV}$, and the $E_{\text {des }}(\mathrm{Ga})$ of $2.5 \mathrm{eV}$. We determine $E_{\text {diff }}$ for In to be $1.6 \mathrm{eV}$ by scaling the $E_{\text {diff }}(\mathrm{Ga})$ of $1.8 \mathrm{eV}$ to bond energies of $\mathrm{GaAs}$ and InAs per bond, i.e., 1.659 and $1.516 \mathrm{eV}$, respectively (see Table I; N.B., InAs and GaAs have four bonds per III-V atomic pair).

\section{Critical cluster size $i^{*}$}

We determine $i^{*}$ in $\mathrm{S}-\mathrm{K}$ mode of InAs islands to be $1-10$ from the $E_{A}$ of $2.0 \mathrm{eV}$ and the $E_{\text {diff }}$ of $1.6 \mathrm{eV}$, using $E_{i *}$ inferred from thermodynamical data and Eq. (1). The common value $\sim 2.0 \mathrm{eV}$ of $E_{A}$ in $\mathrm{S}-\mathrm{K}$ mode is ascribed to the small $i^{*}$.

Now we are ready to discuss $i^{*}$ and $E_{i *}$. From Eq. (1), we have

$$
\frac{E_{i *}+\left(i^{*}+1\right) E_{\mathrm{diff}}}{i^{*}+3}=E_{A} .
$$

Using the $E_{A}$ of $2.0 \mathrm{eV}$ (Fig. 2) and the $E_{\text {diff }}$ of $1.6 \mathrm{eV}$ (Sec. III C), we obtain

$$
E_{i *}=\left(0.4 i^{*}+4.4\right)(\mathrm{eV}) \text {. }
$$

We try to obtain $i^{*}$ from Eq. (3) by assuming an appropriate value of $E_{i *}$.

Let us infer $E_{i} *$ from thermodynamical data. Here we obtain $E_{i *}$ from the heat (enthalpy) necessary to dissociate bulk InAs into single adatoms. We accept $1.6 \mathrm{eV}$ per In-As atomic pair as $E_{i^{*}}$; it is the heat necessary to dissociate InAs(solid) into In(solid) and $\frac{1}{2} \mathrm{As}_{2}$ (gas) (Table I). We assumed that the dissociated state consists of $\operatorname{In}($ solid) and $\mathrm{As}_{2}$ (gas) because the heat of adsorption of $\mathrm{Ga}$ adatoms, 2.5 $\mathrm{eV}$ per atom (Sec. III C), is nearly equal to the heat of sublimation of $\mathrm{Ga}$ (solid), $2.8 \mathrm{eV}$ per atom (Table I). We assumed $\mathrm{As}_{2}$ (gas), not $\mathrm{As}_{4}$ (gas), because the sublimation of GaAs is ascribed to that with the production of $\mathrm{As}_{2}$ (gas) (Sec. III C).
The dissociated state assumed is that in which In is on the surface as adatoms and As is weakly bonded to the surface as $\mathrm{As}_{2}$ (gas).

We can conclude that $i *$ is $1-10$ from the following viewpoints.

First, we determine $i^{*}$ to be 4 assuming the $E_{i *}$ of $1.6 \mathrm{eV}$ per In-As atomic pair. We have $E_{i^{*}}=i^{*} \times 1.6(\mathrm{eV})$ if we assume that the binding energy per In-As atomic pair for an island is the same as that for the bulk. The $E_{i *}$ provides $i^{*}$ of 4 using Eq. (3). Here we neglected surface effects; the $E_{i}$ * obtained is overestimated because an atom on the surface of an island has fewer bonds than a bulk atom.

Second, apart from $1.6 \mathrm{eV}$, we obtain $i^{*}$ of $1-4$ by applying a simple "ball and stick" model to critical clusters. Here we consider a simple model in which the bond energy in islands is the same as that in the bulk and all the In-As atomic pairs in an island have the same number of bonds in the range of 1-4. In the model, the number of bonds per In-As atomic pair, $n_{\text {bond }}$, that are broken in the dissociation of an island into adatoms is 1,2 , or 3 because an adatom should have at least one bond. Because the bond energy in InAs is $1.5 \mathrm{eV}$ per bond, which is obtained from the heat of atomization of $6.1 \mathrm{eV}$ per In-As atomic pair in InAs (Table I), we have $E_{i *}=i^{*} n_{\text {bond }} \times 1.5(\mathrm{eV}) . E_{i *}$ and Eq. (3) give $i^{*}$ of 4 for $n_{\text {bond }}=1, i^{*}$ of 2 for $n_{\text {bond }}=2$, and $i^{*}$ of 1 for $n_{\text {bond }}=3$.

Third, there might be some inconsistency between the experimental results and theoretical expectations if we assume large $i^{*}$. We should consider, from the discussions above, the possibility that $E_{A}$ has a nearly constant value independent of $i^{*}$, based on Eq. (2). The reason is as follows. Assume that $i^{*}$ is large. Then $E_{i *}$ is proportional to $i^{*}$. Here $E_{i *}$ is expressed by $E_{i *}=i^{*} E_{\text {bind }}$, where $E_{\text {bind }}$ is the binding energy of an island per In-As atomic pair; then, we have

$$
E_{A}=\frac{i^{*} E_{\mathrm{bind}}+\left(i^{*}+1\right) E_{\mathrm{diff}}}{i^{*}+3} \rightarrow E_{\mathrm{bind}}+E_{\mathrm{diff}},
$$

when $i^{*} \rightarrow \infty$. In this case, $E_{A}$ has a constant value, and we cannot obtain $i^{*}$ from $E_{A}$. Consider this point. If we assume that $i^{*}$ is large and $E_{\text {bind }}=1.6(\mathrm{eV})$, i.e., $E_{i^{*}}=i^{*}$ $\times 1.6(\mathrm{eV})$, we have $E_{A}$ of approximately $3.2 \mathrm{eV}$ from the $E_{\text {diff }}$ of $1.6 \mathrm{eV}$ and Eq. (4). The $E_{A}$ of $3.2 \mathrm{eV}$ leads to a definitely steeper slope of the Arrhenius plot of the island density than the slope obtained experimentally $\left(E_{A}\right.$ of 2.0 $\mathrm{eV}$ ), as shown in Fig. 1. Hence, there might be inconsistency if $i^{*}$ is large. Consequently, we can reasonably conclude that $i^{*}$ is small.

Now we are ready to state the value of $i^{*}$. We conclude that $i^{*}$ is $1-10$ (here we count In atoms only). Values of $i^{*}$ reported in Refs. 3 and 4 are consistent with the $i^{*}$ determined in the present study.

As mentioned previously, $\mathrm{S}-\mathrm{K}$ mode shows the common value of $\sim 2.0 \mathrm{eV}$ for $E_{A}$ for various materials, thicknesses, and growth conditions (Fig. 2). We obtained the $i^{*}$ of 1-10 from the $E_{A}$ of $2.0 \mathrm{eV}$ for InAs $\mathrm{S}-\mathrm{K}$ islands. We infer, by analogy, that $i^{*}$ 's also have similar values for $\mathrm{S}-\mathrm{K}$ mode. Consequently, we ascribe the common $E_{A}$ to the small $i^{*}$. 
We cannot completely exclude the possibility that actual values of $E_{i *}$ and $E_{\text {diff }}$ are different from those used above. Different $E_{i} *$ and $E_{\text {diff }}$ bring different $i *$.

First, we consider $E_{i *}$. Here we assume that critical clusters are 2D islands because $i *$ obtained above is small. Then $E_{i}$. per atom is the binding energy of an atom at step edge. Itoh et al. reported that the binding energy of a $\mathrm{Ga}$ atom at step edge is $0.25-0.32 \mathrm{eV}$ times $n$ in GaAs growth, ${ }^{31}$ where $n$ is an in-plane coordination number of the atom. We obtain $E_{i *}$ of $0.46-0.58 \mathrm{eV}$ per In atom assuming that the binding energy for adatoms is proportional to that for bulk, as assumed above, and $n=2$. The $E_{i *}$ is notably smaller than 1.6 $\mathrm{eV}$ per In atom used above.

Second, some authors have reported various values of $E_{\text {diff }}(\mathrm{Ga})$. Shitara et al. reproduced results of reflection high energy electron diffraction measurements of GaAs growth by the Monte Carlo simulations. ${ }^{32}$ They reported $E_{\text {diff }}(\mathrm{Ga})$ of $1.58 \mathrm{eV}$. We obtain $E_{\text {diff }}$ of $1.44 \mathrm{eV}$, which is close to $1.6 \mathrm{eV}$ used above, assuming that the binding energy for adatoms is proportional to that for bulk. Thibado et al. measured islands in GaAs growth by scanning tunneling microscopy and reported that $E_{\text {diff }}(\mathrm{Ga})$ is between 1.7 and $2.3 \mathrm{eV}^{33}$ This may lead to a different conclusion if $E_{\text {diff }}(\mathrm{Ga})$ is close to $2.3 \mathrm{eV}$.

Third, Ishii et al. obtained $E_{\text {diff }}$ by the first-principles molecular dynamics calculation and reported that the activation energy for migration of In adatoms on InAs is half of that of In adatoms on GaAs. ${ }^{34}$ If we include the effect, we probably obtain different $i^{*}$.

In conclusion, the number of In atoms in a critical cluster for $\mathrm{S}-\mathrm{K}$ mode of InAs islands, $i *$, was determined to be 1-10 from the activation energy $E_{A}$ of $2.0 \mathrm{eV}$ determined from the Arrhenius plot of the InAs island density. The common $E_{A}$ value of $\sim 2.0 \mathrm{eV}$ in $\mathrm{S}-\mathrm{K}$ mode was ascribed to the small $i^{*}$.

${ }^{1}$ D. Leonard, M. Krishnamurthy, C. M. Reaves, S. P. Denbaars, and P. M. Petroff, Appl. Phys. Lett. 63, 3203 (1993).

${ }^{2}$ J. P. Hirth and G. M. Pound, Condensation and Evaporation (Macmillan, New York, 1963).

${ }^{3}$ R. E. Welser and L. J. Guido, Appl. Phys. Lett. 68, 912 (1996).

${ }^{4}$ Y. Ebiko, S. Muto, D. Suzuki, S. Itoh, K. Shiramine, and T. Haga, Phys. Rev. Lett. 80, 2650 (1998).

${ }^{5}$ K. Shiramine, T. Itoh, S. Muto, T. Kozaki, and S. Sato, J. Cryst. Growth 242, 332 (2002)

${ }^{6}$ A. Madhukar, T. R. Ramachandran, A. Konkar, I. Mukhametzhanov, W.
Yu, and P. Chen, Appl. Surf. Sci. 123/124, 266 (1998).

${ }^{7}$ A. Madhukar, Q. Xie, P. Chen, and A. Konkar, Appl. Phys. Lett. 64, 2727 (1994).

${ }^{8}$ K. Tillmann, D. Gerthsen, P. Pfundstein, A. Förster, and K. Urban, J. Appl. Phys. 78, 3824 (1995).

${ }^{9}$ T. Wang and A. Forchel, J. Appl. Phys. 85, 2591 (1999).

${ }^{10}$ J. Oshinowo, M. Nishioka, S. Ishida, and Y. Arakawa, Appl. Phys. Lett. 65, 1421 (1994).

${ }^{11}$ W. Seifert, N. Carlsson, J. Johansson, M.-E. Pistol, and L. Samuelson, J. Cryst. Growth 170, 39 (1997).

${ }^{12}$ R. Leon, C. Lobo, T. P. Chin, J. M. Woodall, S. Fafard, S. Ruvimov, Z. Liliental-Weber, and M. A. Stevens Kalceff, Appl. Phys. Lett. 72, 1356 (1998).

${ }^{13}$ D. R. Frankl and J. A. Venables, Adv. Phys. 19, 409 (1970).

${ }^{14}$ In Fig. 2, almost all the data points fall around the solid line although the line is a fitted line only for $2 \mathrm{ML}$ InAs/GaAs data $(\bigcirc)$. Although the density for $\mathrm{GaSb} / \mathrm{GaAs}(\times)$ decreases sharply above $520^{\circ} \mathrm{C}$, the authors obtained $2.15 \mathrm{eV}$ for $E_{A}$ from the slope of a fitted line for data below $520^{\circ} \mathrm{C}$ and ascribed the low density above $520^{\circ} \mathrm{C}$ to the enhancement of desorption. The data for $10 \mathrm{~nm} \mathrm{InAs} / \mathrm{GaAs}(\boxplus)$ also give $\sim 2.0 \mathrm{eV}$ for $E_{A}$ although they shift to a lower density than the solid line. Only data for Volmer-Weber growth $(\bullet)$ give $E_{A}$ markedly different from $\sim 2.0 \mathrm{eV}$.

${ }^{15} \mathrm{~K}$. Yagi, Butsuri 28, 108 (1973) (in Japanese).

${ }^{16}$ K. J. Routledge and M. J. Stowell, Thin Solid Films 6, 407 (1970).

${ }^{17}$ M. J. Stowell and T. E. Hutchinson, Thin Solid Films 8, 41 (1971).

${ }^{18}$ D. Leonard, K. Pond, and P. M. Petroff, Phys. Rev. B 50, 11687 (1994).

${ }^{19}$ B. Lewis and D. S. Campbell, J. Vac. Sci. Technol. 4, 209 (1967).

${ }^{20}$ M. J. Stowell, Philos. Mag. 21, 125 (1970).

${ }^{21}$ W. K. Burton, N. Cabrera, and F. C. Frank, Philos. Trans. R. Soc. London, Ser. A 234, 299 (1951).

${ }^{22}$ K. Yamaguchi, K. Yujobo, and T. Kaizu, Jpn. J. Appl. Phys., Part 2 39, L1245 (2000).

${ }^{23}$ T. Haga, M. Kataoka, N. Matsumura, S. Muto, Y. Nakata, and N. Yokoyama, Jpn. J. Appl. Phys., Part 2 36, L1113 (1997).

${ }^{24}$ T. Shitara and T. Nishinaga, Jpn. J. Appl. Phys., Part 1 28, 1212 (1989).

${ }^{25}$ N. Sugiyama, T. Isu, and Y. Katayama, Jpn. J. Appl. Phys., Part 2 28, L287 (1989).

${ }^{26}$ T. Kojima, N. J. Kawai, T. Nakagawa, K. Ohta, T. Sakamoto, and M. Kawashima, Appl. Phys. Lett. 47, 286 (1985).

${ }^{27}$ J. M. Van Hove and P. I. Cohen, Appl. Phys. Lett. 47, 726 (1985).

${ }^{28}$ E. M. Gibson, C. T. Foxon, J. Zhang, and B. A. Joyce, Appl. Phys. Lett. 57, 1203 (1990).

${ }^{29}$ Landolt-Börnstein, New Series, Vol. IV/19A, edited by W. Martienssen (Springer, Berlin, 1999).

${ }^{30}$ Landolt-Börnstein, New Series, Vol. III/17a, edited by K.-H. Hellwege (Springer, Berlin, 1982).

${ }^{31}$ M. Itoh, G. R. Bell, A. R. Avery, T. S. Jones, B. A. Joyce, and D. D. Vvedensky, Phys. Rev. Lett. 81, 633 (1998).

${ }^{32}$ T. Shitara, D. D. Vvedensky, M. R. Wilby, J. Zhang, J. H. Neave, and B. A. Joyce, Appl. Phys. Lett. 60, 1504 (1992).

${ }^{33}$ H. Yang, V. P. LaBella, D. W. Bullock, Z. Ding, J. B. Smathers, and P. M. Thibado, J. Cryst. Growth 201/202, 88 (1999).

${ }^{34}$ A. Ishii, K. Fujiwara, and T. Aisaka, Appl. Phys. Lett. (in press). 